\title{
Attentional Control and other Executive Functions
}

\author{
https://doi.org/10.3991/ijet.v12i03.6587 \\ Maria Karyotaki \\ National Center For Scientific Research "Demokritos", Athens, Greece \\ karyotakimariaegmail.com \\ Athanasios Drigas \\ National Center For Scientific Research "Demokritos", Athens, Greece \\ dr@iit.demokritos.gr \\ Charalabos Skianis \\ University of the Aegean, Karlovassi, Greece \\ cskianis@aegean.gr
}

\begin{abstract}
Current article aims to shed light on the reciprocal relation between attentional control and emotional regulation. More specifically, there is a verified relation between attention and cognitive, metacognitive and emotional processes, such as memory, perception, reasoning as well as inhibitory control, cognitive flexibility, self-monitoring and positive moods. In addition, positive mood has been already reciprocally related to a broad attentional scope as well as to an increased cognitive flexibility. Future research should focus on the effects of attentional control on cognitive control processes, thereby, on individuals' emotional regulation, as a whole. Evidently, an advanced research in the relation of attentional control and emotional regulation could develop a comprehensive methodology for counterbalancing the difficulties facing individuals with ADHD, Autism Spectrum Disorder, Oppositional Defiant Disorder or even depression.
\end{abstract}

Keywords - executive functions, attention, Attention Deficit Hyperactivity Disorder (ADHD), self-consciousness

\section{$1 \quad$ Introduction}

"The definition of executive function usually includes the concept of controlling attention, mental flexibility, goal-directed behavior and the ability to anticipate the consequences of one's own behavior. Moreover, the concept of self-awareness and the idea that the frontal lobes serve as a manager and programmer of human psychological processes (metacognition) are also included in this definition (Ardila, 2016)".

According to Ardila, there are two distinct subgroups constituting individuals' executive functioning; Metacognitive as well as Emotional/Motivational executive functions. Metacognitive executive functions refer to response inhibition, conflict monitoring and switching, self-awareness, temporality of behavior, self-consciousness, 
working memory, abstraction and problem solving. Emotional/motivational executive functions entail the coordination of cognition and motivation as well as the ability to control emotions and behavior.

The present article attempts to bring forward the importance of the executive component of attention in both individuals' metacognitive and emotional regulation processes. Thus, attention could become the common grounds on which individuals' executive functioning could be improved in a systemic and holistic way.

\section{Attentional control and metacognitive executive functions}

Strobach et al. showed that experienced video gamers acquire optimized and speeded executive control processes as they coordinated two different tasks either simultaneously in dual-task situations with novel stimuli or stimulus-response mappings or sequentially in task switching tests. The researchers made two experiments in order to investigate video game trainees' improved executive control skills in an attempt to select and execute multiple responses. The first experiment taken place, involved a dual-task test consisting of a single-task situation and a series of dual-task block trials with the simultaneous presentation of auditory and visual stimuli. The second one, constituted a task switching test with single and mixed-task blocks, wherein predictive switches occurred between task-switch trials and repeat trials. Both tests were performed before and after video game practice. A scatter plot of the Brinley-plot type demonstrated action video gamers' superior executive control skills in complex situations calling for an effective regulation of the switch between conflicting stimuli in intermixed task settings. It seems that executive control skills, such as attentional control can be improved, thus become traceable when a person coordinates different tasks involving simultaneous or sequential, but certainly rapid switches between them.

Keil et al. describes executive processes as a prerequisite function for individuals' adequate response to novel and complex environmental demands. The former consist of several cognitive and metacognitive processes, such as self-monitoring, selfawareness and self-regulating, inhibiting irrelevant stimuli, shifting between concepts or actions, generation and application of strategies, temporal integration of information as well as recruiting or integrating multimodal inputs from throughout the brain. More specifically, researchers compiled an annotation of neuropsychological tests for the purpose of identifying the abilities that underlie executive functions and therefore discriminate cognitively impaired patients. Neuropsychological experiments should encompass multiple measures or administration of control tasks in addition to executive-level tasks so as to discern the executive functions from other contributing factors, such as basic or focal processes. Overall, control tasks should tap visuospatial, attentional or other processes likely to be involved in respective executive function tests. Moreover, the cognitive processes addressing participants' planning, scheduling, strategy use and rule adherence compose the first set of executive function tests. Two of these tests (the Tower of Hanoi and the Tower of London) are computerized. In addition, executive control processes, such as generating word or de- 
signs without repetition and complying with environmental constraints, are subject to monitoring skills and can be measured by fluency and generation tests. The third category of tests is centered on sustaining or/and shifting attention and suppression of habitual responses through self-regulating one's behavior. Finally, tests of concept formation and abstract reasoning, requiring continuous monitoring and modulation of output tap of another domain of executive functions. As many of the aforementioned tests deploy linguistic processes or language, the relation between executive functions and verbal intelligence via internal speech or verbal mediation in language-disordered population, should be clarified.

Mertes et al. investigated on the effects of spatially and temporally irrelevant information on the processing of subsequent information. Relevant information is maintained in visual working memory and its representations facilitate the adaptability of the human behavior. Notwithstanding, visual attention is captured by irrelevant objects in the visual field. Thus, these automatic, involuntary shifts of attention occur due to distracting salient stimuli or stimuli that match the representations held in visual working memory. As a result, suppression mechanisms are activated, the attentional control sets, to re-orientate and reallocate one's attention to the sought-after objects. Moreover, it has been found that individuals' responses at spatial cuing tasks are faster when the target and the preceding cue shared the same location. Attention was already drawn to the location of the cue, thereby reinforcing the processing of the object, subsequently presented at that location. In the present study, an irrelevant color cue that was either contingent (color search) or non-contingent (shape search) on attentional control sets was presented, prior to a target array with different stimulus-onset asynchronies (SOA). More specifically, cue displays containing a lateralized color singleton were presented prior to the target display. Participants were instructed to ignore the task-irrelevant cue display as the cue position did not allow for predicting the target position. In one condition, both cue and target singletons were defined by color (contingent condition). In the non-contingent condition, color singleton cue and target singleton did not share features in order to facilitate the inhibition of the color singleton cue. Researchers measured event-related potentials (ERPs) evoked by the cue display. The findings of the study sum up to two independent filtering stages regarding the passage of information into working memory. First, an early selection process represented by an $\mathrm{N} 2$ posterior contralateral (N2pc) to the singleton cue and a component labeled distractor positivity (Pd-early), enables the active re-orienting of the attentional focus in the search array when irrelevant information matches working memory templates. Second, there is a more conservative filtering stage determining visuo-spatial working memory access, associated with Contralateral delay activity (CDA) and distractor positivity (Pd-late) component, which encompasses the active maintenance of working memory representations surviving after the first inhibition of the cued location as well as the final inhibition of the false representations as soon as the relevant information is obtained. At that point individuals are able to proceed with the rapid perception of the target. Therefore, attentional processes seem to be strongly linked to cognitive control mechanisms, such as the distractor positivity ( $\mathrm{Pd}$ ) component that assists people to prioritize their targets. The distractor positivity (Pd) component has been found to be subjected to exterior incentives, such as rewards. Fur- 
thermore, individuals' attentional mechanisms through the manipulation of their distractor positivity $(\mathrm{Pd})$ component are adaptable and susceptible to motivation.

Anderson conceptualizes executive function as an integrated supervisory or control system, made up of multiple process-related systems. More specifically, attentional control processes include the capacity to selectively attend to specific stimuli and inhibit prepotent responses as well as the ability to focus attention for a prolonged period. In advance, individuals' acquiring attentional control involves the regulation and monitoring of actions in order for them to complete tasks, avoid procedural mistakes and respond appropriately. Furthermore, information processing can be evaluated by the speed, quantity and quality of output. Cognitive flexibility, including the working memory capacity, refers to the ability to shift between response sets, learn from mistakes, devise alternative strategies, divide attention and process multiple sources of information concurrently. Finally, goal setting lays on individuals' ability to develop new initiatives and concepts while planning actions to approach tasks in an efficient and strategic manner. Effective assessment of children's executive function is quite challenging due to the need for capturing quantitative factors as well as cognitive-related processes and behavioral elements. Moreover, individuals' executive control reaches its maturity when the respective cognitive processes are fully established around mid-adolescence or early adulthood. Therefore, longitudinal studies will be required so as to verify any conceptualization concerning executive function development.

Davidson et al. made a comparative study on children, adolescents and adults' cognitive control skills and executive functions by measuring the interrelations and developmental progressions of their working memory, inhibitory control and task switching skills. Participants were assessed through a computerized battery of 4 related tests for about $30 \mathrm{~min}$ in total. The first test was a classic Simon task, where the stimuli were presented randomly on the left or right of the screen over the block of 20 trials. In the second test, a single large arrow pointed straight down and participants had to respond on the same side as the arrow in a randomized mixed block of 20 congruent and incongruent trials at the left or right of the computer screen. In the Dots test, a large dot was presented either at the left or right on each trial. The two types of dots used were either striped or solid, which indicated that when participants saw the striped dot they would have to make a response towards the same side, whereas when they saw the solid dot they would have to respond on the side opposite the dot. An initial block of 20 congruent trials was combined with a block of 20 incongruent trials as well as a block of 20 randomly intermixed trials. In the last test, each stimulus, an abstract shape, was presented in the center of the rectangle. For each stimulus participants were taught a rule and there were two conditions involving two- or six- abstract shapes. They first completed 2 blocks of 20 trials of the two-shape condition accompanied by 2 blocks of 20 trials for the six-shape condition. The dependent measures were accuracy of responses, speed/reaction time (RT) and percentage of anticipatory responses (AR). The results verified researchers' hypothesis that the cost of exercising inhibition to respond faster and more accurately on spatially incompatible trials (the Simon effect) decreased from 6 years of age onward. In regard to the integration of arbitrary rules related to the stimulus of each trial with the location of the stimulus, 
the spatial incompatibility effect decreased significantly from age 6 onward in accuracy, but only on the Arrows test. On the whole, participants' performance was slower and less accurate on switch than nonswitch trials in both the Arrows task and the Dots-Mixed condition. However, the response time did not change between children of 6-13 years and adults on both arrows and Dots-Mixed condition due to the univalent stimuli employed in a task or rule. Furthermore, accuracy and impulsivity differences between the Dots-Incongruent and Dots-Congruent conditions decreased over age. Notwithstanding, on both the Arrows test and the Dots-Mixed condition adults and older children showed greater speed response cost for switching to the Congruent rule. Furthermore, adults' response time decreased when both the rule and responsesite switched. However, the youngest children of 4-8 years performed better in accuracy and reaction time on switch trials where the response-site remained the same. Therefore, cognitive flexibility in an intermixed, task-switching context, characterized by the "all or none" principle where participants inhibit a dominant response all the time, was evident in participants with mature cognitive system, after 13 years of age. The last finding indicates that adult participants adjusted their speed to preserve accuracy on the more difficult trials. On the contrary, young children were often too impulsive to take the time they needed at the cost of accuracy. Moreover, adults seemed to be able to reset their default response between the two conditions of the Mixed block in order to exercise inhibition in a steady state. Furthermore, the study aimed to delineate the interactions among the basic executive processes, such as inhibitory control, memory and task switching. As far as the inhibitory control is concerned, the ability to inhibit attention to distractors or the ability to inhibit a strong behavioral inclination is tied to a person's cognitive flexibility. Consequently, selective and sustained attention is critical for adaptability and creativity in the way people hold and manipulate information. When the tasks were similar in difficulty, participants' speed on working memory and inhibition were highly correlated, whereas their accuracy on working memory and inhibition was respectably correlated.

Pureza et al. present the relationship between executive functions, such as the central executive of working memory, inhibition, cognitive flexibility and selfmonitoring in late childhood. The instruments deployed were the Unconstrained, Phonemic and Semantic Verbal Fluency subtests of the Montreal Battery of Evaluation of Communication, the Random-Number Generation task, the Bells Test, the nback Test and the Hayling Test. The results suggest a relationship between inhibitory control, cognitive flexibility, processing speed as well as the central executive of working memory. More specifically, a strong association was brought into light between language alternation as an aspect of cognitive flexibility and inhibitory control as the ability to inhibit responses unrelated to the context. Likewise, selective attention and processing speed in verbal fluency were also related. On the one hand, attention, inhibition and verbal-semantic linguistic processing and on the other hand, focused attention, inhibition, verbal processing speed and self-monitoring were found to be linked. Furthermore, inhibition and cognitive flexibility of semantic verbal fluency were related to the central executive component of working memory. Regression analysis on larger samples could verify and expend current findings. 
Rudkin et al. inquired into the relation between the visual-spatial and central executive components of working memory through a triplet of dual-task coordination experiments. The central executive is responsible for complex functions, such as comprehension, reasoning, strategic cognitive control and dual-task coordination. Both visual and spatial working memory tasks, the Matrix Patterns and the Corsi Blocks respectively, were used as a means for discriminating the function of visual and spatial working memory subcomponents. The dynamic role of the spatial component is apparent as it records and encodes information about movements and sequences of movements as well as it entails one's inner ability to monitor and control his spatial attention. Thus, there seems to be an interrelation between one's executive functioning and his visual-spatial processes, which is worthy to be looked into. In the first experiment, central executive processes, such as information updating, attention shifting and inhibiting inappropriate information were more closely related to the spatial component than the visual component in a dual-task condition. In this case, both the Matrix Patterns task and the Corsi Blocks task were combined concurrently with an oral random generation task. Participants' performance in the spatial task decreased much more than their performance in the visual task, indicating the former's tapping of executive processes, although there was no significant difference in random generation performance between the two dual-task conditions. In the second experiment, researchers investigated the effect of a dual-task condition experiment combining the concurrent presentation of an oral random generation task and a visualspatial task, either with sequential or simultaneous stimuli. The results of the second experiment verified the assumption that the recall of both item and order information in a serial sequential presentation taps of central executive functioning more than in simultaneous visual-spatial tasks. Finally, the third experiment controlled for the interference of short-memory in the random generation task, thereby minimizing its influence on the measurement of executive functioning. The two previous visualspatial tasks were concurrently combined with either random- and fixed-interval auditory tone repetition tasks. More specifically, the fixed-interval tone task was expected to load the executive to a lesser extent than the random-interval task. However, both tone tasks interfered with the sequential presentation task only and the disruption caused by the two tone tasks was not significantly different. Overall, a serialsequential visual-spatial task taps of executive processes to a greater extent than simultaneous item information presentation tasks. This is because sequential presentation may lead to a significant increase in one's demand of strategic monitoring and control over the encoding and rehearsal of the mental path configurations from the movement patterns of the stimuli. The question remains which part of the sequential task, encoding, maintenance or retrieval, places greater executive load.

Yates et al. made a correlational study between executive processes and other functions, such as working memory, the executive attention construct and prospective memory. In this study, it is insinuated that shifting, inhibition and updating are three independent, but interrelated subprocesses compiling one's executive function. Shifting corresponds to attention switching or task switching. Inhibition is the intentional and controlled suppression of dominant, automatic and prepotent responses. Updating lays on renewing and monitoring the working memory representations. Previous stud- 
ies considered executive functions as a fraction of goal directed behavior, attention control, temporal organization and planning. In the current study, the Wisconsin Card Sorting Test was implemented as a means for measuring participants' cognitive flexibility and problem solving skills. In addition, the Brazilian Brief Neuropsychological Assessment NEUPSILIN measured participants' time and spatial orientation, sustained auditory attention, visual perception, memory, arithmetic abilities, language skills, motor abilities and two subcomponents of executive functions, such as solving simple problems and verbal phonemic-spelling fluency. The study aimed at relating one's performance in the WSCT to one's performance in the cognitive tasks encompassed in the NEUPSILIN. The results verify the existence of the executive attention construct that is the ability to keep an objective in active state during the task and the ability to overcome interference, particularly in a conflicting situation. Sustained attention, prospective memory and verbal fluency were moderately correlated in tests as well as executive functions correlated with episodic-semantic memory and oral and written language. Furthermore, one's executive function was related to one's working memory capacity. WCST entails both visual and oral language stimuli for the tapping of abstraction, insight formation and mental flexibility, thus depicting the multimodality of the measure. Nevertheless, more implicit language recognition demands in the tasks would deliver greater language inferential processing associations by virtue of the close relation between visual-spatial information and executive functioning or attentional control.

Rhodes et al. investigated both the direct and indirect relation between executive functions and individuals' memory accuracy in terms of the latters' capacity to recall and monitor correct information. As executive functions decline with age, older adults exhibit low performance both in measures of their memory accuracy and memory monitoring. More specifically, older adults' ability to encode and retrieve information as well as their ability to monitor their feeling-of-knowing judgments diminishes, respectively. Notwithstanding, between executive functions and memory monitoring lays the quality of information available in one's memory. In the current study, there were two recall stages, either the forced recall or the free recall stage. In the forced recall stage participants had to provide as many correct responses as possible and in the free recall stage participants had to control the accuracy of their responses through calling the probability of being correct while setting a response criterion. If the probability of being correct exceeds or is equal to the response criterion, an answer is volunteered; otherwise the response is withheld. Control processes influence accuracy by determining whether a response is volunteered or withheld. Therefore, the free recall stage encompasses three criterions in total; Monitoring effectiveness, control sensitivity and the respondents' incentive towards their accuracy estimation. Speed of processing and working memory was separately measured. The measured deployed in the study were: The Digit-Symbol Substitution Task, the Number Comparison Task and the Trail Making Test, the computerized version of the WCST, the Controlled Oral Word Association test and the Working Memory OSPAN task. The findings verified that memory accuracy, quantity of correct items retrieved and executive function were found to be directly related to age. Executive functions affected memory accuracy directly through their contribution to the quantity of correct responses available at the 
forced recall stage and indirectly through the monitoring of deceptive items at the free recall stage. However, memory monitoring partially mediated the quantity of the correct responses at the free recall stage for deceptive items, but not for control items. Overall, participants' accuracy of retrieved responses and memory monitoring can predict their memory accuracy. Furthermore, the relation between the specific components of executive functions in their full range and memory accuracy measures as well as the possible interplay between retrieval and monitoring processes are yet to be found.

Salvi et al. made a review on how eye movements and blinking relate to different types of cognitive processes, such as focus of attention and memory search through the implementation of the eye movement monitoring technique. More specifically, research has shown that spontaneously looking at a blank area and positioning the eyes on a location congruent with the location of stimuli during encoding facilitates retrieval. Moreover, eye movements predict recall performance of visual and conceptual information, even after the stimuli are no longer present. Attention is tightly linked to eye movement by programming explicit ocular movements. External (bottom-up) attention is responsible for selecting and modulating of sensory information, specific in spatial location and time, whereas internal (top-down) attention operates over information with a pre-existing internal representation. These types of attention are mutually exclusive and interact with cognitive control processes responsible for attention shifting. Decreased blinking and increased number of fixations correspond to externally focused attention in contrary to internally focused attention processes that include increased blinking, decreased eye movements and increased fixations on empty space. Therefore, influencing eye movements and blink rates might influence attention and memory retrieval.

Schneider et al. highlighted the multiple roles of attentional processes in visual perception, memory and sensorimotor control. This article focuses on eye movements, an overt index of visual selection and their relation to covert shifts of attention involved in competition and priority control. More specifically, tracking eye movements and measuring gaze allocation through implementing powerful wearable eye trackers and virtual reality technology shall prepare the ground for a solid theoretical and modeling framework around attentional processes in real-world tasks. It seems that there is a pre-attentive coding of action selection processes, following a top-down suppression of potential targets without current interest, evident in a proactive gaze behavior. In addition, task-relevant items and their position can be memorized better if the observer is actively engaged in the task. Position can also be memorized well if the observer is moving through the real-world setting without manipulating the objects. Notwithstanding, gaze allocation preference towards task-relevant items and their recall require active object manipulation. Therefore, memory modulates current perceptual and attentional processing.

Kunda et al. presented an innovative computational model using the Embedded Figures Test (EFT) that relates variations in sequences of attention to stored memory representations of target items. This widely used neuropsychological assessment requires searching for a simple geometric figure within a larger, more complex figure. Moreover, internal access to these representations can affect overall, visual search 
performance. Thus, the target template originating from visual working memory is itself subjected to attentional deployments. However, this model did not predict from the very beginning the optimum anchor features that define the initial saliency map, thereby future studies should focus on how artificial agents, just as human subjects, choose such features.

Bridewell et al. introduced a core theory of attention, encompassing vision, audition, reasoning and working memory in combination with a computational system implementing that theory, "Arcadia". This cognitive system and computational model is structured on the assumption that a modality independent mechanism of attention plays a central, integrating role for perception, cognition and action. The current system detects and tracks objects in dynamic environments so that it proceeds incrementally from sensory signals to encoded representations in working memory. In "Arcadia" attentional strategies prioritize interlingua elements by name, type and potentially other fields. Every particular task involves supplying a specific strategy with its own prioritization routine, driven by the system's deployment of attention either to preattentive or task-related information. Without task, "Arcadia" relies on a default strategy lacking in a specific attentional strategy. Moreover, "Arcadia" can produce integrated representations of objects or other content as well as it can distinguish between information encapsulated within components and information made available to all components (conscious access). Furthermore, "Arcadia" may be used as a cognitive system for modeling the effects of the deployment of attention, outward and/or inward, on individuals' cognitive control and by far, on their emotional regulation.

\section{Attentional control and emotional/motivational executive functions}

Petrovic et al. presented an integrated model of mechanistically related processes, either emotional or non-emotional, tied to the dysfunctional top-down regulation of information processing in ADHD and emotional instability disorders. Top-down regulation refers to the reciprocal interaction of information between multiple regions in large-scale, emotional and non-emotional networks with hierarchical perceptionaction organization. Thus, the highest level of emotional and non-emotional processes performs the more complex and temporally dispersed information. Moreover, topdown control of the emotional and the non-emotional systems vary in their efficiency among the general population. In addition, ADHD patients have been associated with poor non-emotional top-down control and individuals with emotional instability disorders have been associated with poor emotional top-down control. Therefore, patients' with ADHD would show a dysregulation in the non-emotional system and normal emotional regulation, whereas individuals with emotional instability disorders would show a dysregulation in the emotional system and normal non-emotional regulation. Comorbid states may encompass poor capacity for both non-emotional and emotional processing. However, the aforementioned dysregulation shares common grounds on account of an underlying neuromodulatory mechanism, the dopamine system, which interacts with both emotional and non-emotional networks. Its proper 
treatment would affect the whole regulatory brain network, processing either emotional or non-emotional types of information.

Francesco et al. reviewed on the executive function deficits in Attention- Deficit/Hyperactivity Disorder (ADHD) and Autism Spectrum Disorder (ASD). Research on the executive function deficit directly associated with the aforementioned groups lays on the latter's shared genetic and neurobiological underpinnings, leading to a disturbance at the level of the executive functions. Moreover, the co-occurrence of ASD and ADHD could inaugurate a combined phenotype, reflecting an additive comorbidity, thus augmenting enhanced treatment options. Executive functions, such as planning, spatial and verbal working memory, response inhibition and vigilance have been linked to ADHD children/adolescents. Moreover, an ADHD subtype, the ADHD-I has been related to sluggish, disorganized behavior. Children with ADHD also have deficits in sustained attention and visual memory. Likewise, constant impairments in cognitive flexibility, planning and working memory have been found in individuals with high-functioning autism. In addition, children with autism have shown more deficits in shifting attention, sustained or selective attention and response inhibition. Furthermore, a clinical combined phenotype, ASD+ADHD, was attempted to be investigated separately regarding the level of the following executive functions: inhibition, working memory, flexibility, attention, planning, monitoring, preparatory processing, fluency and concept formation. Attention was found to be dysfunctional in all clinical groups (ASD, ADHD and ASD+ADHD). The ADHD children had a deficit in the manipulation of sustained and divided attention compared to healthy children, whereas children with ASD+ADHD performed worse in divided attention and alertness tasks compared to their healthy counterparts. In contrast, both ASD and ASD+ADHD groups were found to be lacking in cognitive flexibility and planning. The ASD was particularly related to cognitive flexibility deficit and the ASD+ADHD group was found to be impaired in duration concerning both flexibility and planning tests. ADHD and ASD+ADHD groups were also lacking in response inhibition. Consequently, cognitive training in executive functions may change the underlying neural mechanisms to improve the above groups' real-world function.

Jiang et al. explored emotional regulation and executive functions in the Oppositional Defiant Disorder (ODD) and in ADHD children. Additionally, the relationship between emotional regulation and executive functions were intrinsically investigated. In the dual-pathway model of executive functions, attention, working memory, planning and response inhibition pertain to cold executive functions in comparison with the hot executive functions embracing one's emotional and motivational regulation. Although ADHD children have been related to most executive function deficits, both cold and hot, ODD children have been connected mainly to deficits in individuals' visual working memory and response suppression/inhibition. The present study is particularly interested in the relation of ODD and ADHD children with emotional regulation as part of executive functioning, subjected to motivations and emotions. The Adolescent Daily Emotional Regulation Questionnaire (ADERQ), the computerized version of the Wisconsin Card Sorting Test (WCST), the Wechsler Intelligence Scale for Chinese children (WISC-IV), the Stroop color-word association test and the Cambridge Neuropsychological Test Automated Battery (CANTAB) were the re- 
search tools deployed. The findings of the study verified that ODD children face deficits in emotion regulation processes, albeit their exact behavioral characteristics as well as their whole executive functioning profile and the role of the comorbidity with the ADHD syndrome in their emotion dysregulation remains unclear. Notwithstanding, the ability to regulate emotions is an important part of an individual's executive functions. In this case, ODD children were prone to greater number of perseverative errors due to their lack in self-reflection, self-control and self-regulation of negative emotions combined with deficits in working memory and planning capabilities. On the whole, response inhibition incapability, planning dysfunction and emotion dysregulation were important risk factors for ODD children.

Van Stralen made a review study on the relation between children's emotional dysregulation and attention-deficit/hyperactivity disorder. Emotional dysregulation stands for one's reactive or motivational control problems leading to hyperactivityimpulsivity symptoms due to inappropriate internalized (sadness, depression) or externalized (anger, aggressiveness) emotional responses. More specifically, the emotional dysregulation in ADHD refers to individuals' lack in a whole set of cognitive and meta-cognitive skills, such as attention, self-control, self-regulation and selfmonitoring. Furthermore, emotional impulsiveness as well as deficient self-regulation of affections-motivation-arousal, internalization of speech and reconstitution in the ADHD syndrome co-occurs with other executive functions, such as inhibition and working memory. Latter research illustrated that ADHD individuals' behavioral dysregulation and difficulties in metacognition are interconnected. Thus their emotional dysregulation and deficits in executive functions can be interdependent and all contribute independently to distinguishing between children with ADHD and controls. Further research on the field of emotional dysregulation in ADHD individuals could offer a crystallized definition of the construct as well as a comprehensive methodology for building innovative training and assessment tools.

Vanlessen et al. introduced an alternative theoretical framework for mood-related tuning of spatial attention encompassing the role of cognitive control and flexibility on mood-attention interactions. Research has shown that mood can dynamically modulate attentional control either through increasing baseline activation of the visual cortex and thus, boost external stimuli processing or through triggering a deployment of resources to internal information processing at the expense of processing other stimuli. Furthermore, affective state alters monitoring of attentional resources allocation between external and internal attention through the reciprocal relation between the, inherently, rewarding broadening of attentional scope and positive mood. Nevertheless, prefrontal-based cognitive control and flexibility processes take over when a task is demanding, thus reducing resources towards outward attention in a proactive way. In addition, control processes tend to swift attention in a reactive way, if the task is less demanding and even more, when the individual has a positive mood. Therefore, mood and cognitive control processes interact dynamically to determine whether inward or outward attention prevails. 


\section{Conclusions}

Attentional control processes are multimodal and encompass the ability to reach and maintain a vigilance state (alerting system), the ability to focus attention for a prolonged period to specific, mainly, visuo-spatial stimuli (orienting system) as well as the ability to inhibit prepotent responses and regulate/monitor one's actions (executive component or conflict resolution system) (Sperduti et al., 2016).

In order to enhance the diagnostic utility of executive function measures, such as attentional control, integrated models that combine perception, memory and reasoning should be implemented. Artificial intelligence tests of executive functions should integrate internal and external deployments of attention with several other mental processes, such as visual working memory, memory monitoring as well as emotional regulation processes.

Attentional control processes seem to underlie the whole construct of executive functioning as they are strongly linked to individuals' cognitive and metacognitive control processes as well as flexibility mechanisms, such as the inhibitory control and task prioritization (Vanlessen et al., 2016; Zendel et al., 2016; Marzocchi, 2006). Broadening of attention and increased flexibility of cognitive functioning has already been related to positive mood (Fredrickson, 2001; Xu et al. 2015)

Since higher-order cognitive control processes interact dynamically with one's mood state for allocating his attention towards internal or external stimuli in the most plausible way, it should be worthy investigating on the reciprocal relation between attentional control and control processes as well as their effect on individuals' emotional regulation. In addition, a broad attentional scope towards external stimuli has been reciprocally connected to positive mood as detecting information and acquiring knowledge is, inherently, rewarding.

What's more, Ardila stated that individuals' attentional control processes are primary (they emerge by the child's 1st year), include meta-cognitive and motivational/emotional control mechanisms and lead to improvements in the ability to inhibit overlearned behavior (inhibitory control of behavior). In this sense, attentional control could improve individuals' emotional regulation throughout their lives, as well.

Therefore, attentional control and its inspected relation to emotional regulation processes, could substantially assist individuals with ADHD in controlling their emotions and the brain's reward systems, thus their sensitivity against reinforcement and loss (Skogli et al., 2013). Also, the eminent role of emotion regulation in individuals with ADHD is described under the umbrella disorder known as reward deficiency syndrome (RDS) (Blum et al., 2008). As proposed by multiple pathway models in ADHD and Tourette's Syndrome, emotional and motivational regulation processes may constitute another promising endophenotype (Sonuga-Barke, 2003, 2005), explaining ADHD symptoms, such as anxiety and depression (Hovik et al., 2015).

Overall, by establishing a reciprocal connection between individuals' attentiomal control and emotional/motivational regulation, individuals will be benefited in multiple ways: first, holistic therapies can be adopted in improving children's and adolescents' with ADHD cognitive and social behavior (Bunford et al., 2015) as well as enhancing individuals' cognition and behavior, in general (Vanlessen, 2016). Second, 
the interweaving of metacognitive and emotional/motivational executive functions shall proclaim the need for discovering one's own "self-consciousness" (Posner et al., 1998 ) in order to improve his behavior; and "self-consciousness" refers to the interdependence between one's cognition/metacognition and his inner emotions or even further, the interdependence between one's cognition/metacognition and his social emotion (theory of mind).

\section{$5 \quad$ References}

[1] Ardila, A. : "Is self-consciousness" equivalent to "executive function?", Psychology and Neuroscience, pp. 1-6, (2016). https://doi.org/10.1037/pne0000052

[2] Ardila, A. : "Development of Metacognitive and Emotional Executive Functions in Children”, Applied Neuropsychology: Child, pp. 1-6, (2013).

[3] Strobach, T. Frensch, P. A. and Schubert, T. : "Video game practice optimizes executive control skills in dual-task and task switching situations", Acta Psychologica, vol. 140, pp. 13-24, (2012). https://doi.org/10.1016/j.actpsy.2012.02.001

[4] Keil, K.and Kaszniak, A. W. : "Examining executive function in individuals with brain injury: A review”, Aphasiology, vol. 16, (3), pp. 305-335, (2002). https://doi.org/10.1080/ 02687030143000654

[5] Mertes, C. Wascher, E. and Schneider, D. : "From capture to inhibition: How does irrelevant information influence visual search? Evidence from a spatial cuing paradigm, Frontiers in Human Neuroscience, vol. 10, (232), pp. 1-13, (2016).

[6] Anderson, P. : "Assessment and Development of Executive Function (EF) during Childhood", Child Neuropsychology, vol. 8 (2), pp. 71-82, (2002). https://doi.org/10.1076/ chin.8.2.71.8724

[7] Davidson, M. C. Amso, D. Anderson, L. C. and Diamond, A.: "Development of cognitive control and executive functions from 4 to 13 years: Evidence from manipulations of memory, inhibition, and task switching”, Neuropsychologia, vol. 44 (11), pp. 2037-2078, (2006). https://doi.org/10.1016/j.neuropsychologia.2006.02.006

[8] Pureza, J. R. Jacobsen, G. M. Oliveira, R. G. and Fonseca, R. P. : "Relationships between executive function tasks in late childhood", Psychology \& Neuroscience, vol. 4, (3), pp. 369-376, (2011). https://doi.org/10.3922/j.psns.2011.3.010

[9] Rudkin, S. J. Pearson, D. G. and Logie, R. H. : "Executive processes in visual and spatial working memory tasks", The Quarterly Journal of Experimental Psychology, vol. 60, n. 1, pp. 79-100, (2007). https://doi.org/10.1080/17470210600587976

[10] Yates, D. B. Zibetti, M. R. Pawlowski, J. Salles, J. F. De Mattos Pimenta Parente, M. A. De Lima Argimon I. Fonseca, R. P. and Trentini, C. M. : "WCST and NEUPSILIN: Relationships among Executive Functions, Attention, Memory and Language", Psicologia: Reflexao e Critica, vol. 26, (3), pp. 506-515, (2013). https://doi.org/10.1590/S010279722013000300010

[11] Rhodes, M. G. and Kelley, C. M. Executive processes, memory accuracy, and memory monitoring: An aging and individual difference analysis", Journal of Memory and Language, vol. 52, pp. 578-594, (2005). https://doi.org/10.1016/j.jml.2005.01.014

[12] Salvi C. and Bowden E. M. : "Looking for creativity: Where do we look when we look for new ideas?", Frontiers in Psychology, vol. 7 (161), pp. 1-12, (2016). https://doi.org/10.3389/fpsyg.2016.00161 
[13] Schneider W. X. Einhauser W. and Horstmann G.: "Attentional selection in visual perception, memory and action: a quest for cross-domain integration", Phil Trans R Soc B, vol. 368, pp. 1-6, (2013). https://doi.org/10.1098/rstb.2013.0053

[14] Kunda M. and Ting J. : "Looking around the Mind's Eye: How internal deployments of attention can affect visual search performance", Proceedings of the Third Annual Conference on Advances in Cognitive Systems, article 3, pp. 1-16, (2015).

[15] Bridewell W. and Bello P. F. : "A theory of attention for cognitive systems", Advances in Cognitive Systems, vol. 4, pp. 1-16, (2016).

[16] Petrovic, P. and Castellanos, F. X. : "Top-down dysregulation-From ADHD to Emotional Instability", Frontiers in Human Neuroscience, vol. 10, (70), pp. 1-25, (2016). https://doi.org/10.3389/fnbeh.2016.00070

[17] Francesco, C. Francesco, M. Legrottaglie, A. R. Palumbi, R. De Giambattista, C. and Margari, L. : "A review of executive function deficits in autism spectrum disorder and attention-deficit/hyperactivity disorder", Neuropsychiatric Disease and Treatment, vol. 12, pp. 1191-1202, (2016).

[18] Jiang, W. Li, Y. Du, Y. and Fan, J. : "Emotional regulation and executive function deficits in unmedicated Chinese children with Oppositional Defiant Disorder", Psychiatry Investigation, vol. 13, (3), pp. 277-287, (2016). https://doi.org/10.4306/pi.2016.13.3.277

[19] Van Stralen J. : "Emotional dysregulation in children with attention-deficit/hyperactivity disorder", ADHD Attention Deficit Hyperactivity Disorder”, pp. 1-13, (2016). https://doi.org/10.1007/s12402-016-0199-0

[20] Vanlessen N. De Raedt R. Koster E. H. W. and Pourtois G. : "Neuroscience and Biobehavioral Reviews", vol. 68, pp. 816-837, (2016). https://doi.org/10.1016/j.neubiorev. 2016.07.001

[21] Sperduti, M. Makowski, D. and Piolino, P. :"The protective role of long-term meditation on the decline of the executive component of attention in aging: a preliminary crosssectional study", Aging, Neuropsychology and Cognition, 23 (6), 691-702, (2016). https://doi.org/10.1080/13825585.2016.1159652

[22] Zendel, B. R. De Boysson C. Mellah S. Demonet J.-F. and Belleville S. : "The impact of attentional training on event-related potentials in older adults", Neurobiology of Aging, vol. 47, pp. 10-22, (2016). https://doi.org/10.1016/j.neurobiolaging.2016.06.023

[23] Marzocchi, G. M.: "Executive Function and Attention Profiles of Children with ADHD and/or Reading Disorder: Developmental Neuropsychology and Genetic Contributions", (2006).

[24] Fredrickson B. : "The role of positive emotions in positive psychology: the broaden-andbuild theory of positive emotions", Am. Psychol., vol. 56 (3), pp. 218-226, (2001). https://doi.org/10.1037/0003-066X.56.3.218

[25] Xu Y. Yu Y. Xie Y. Peng L. Liu B. Xie J. and Li M. : "Positive affect promotes well-being and alleviates depression: the mediating effect of attentional bias", Psychiatry Res., (2015). https://doi.org/10.1016/j.psychres.2015.06.011

[26] Skogli, E. W. Egeland, J. Andersen, P. N. Hovik, K. T. and Øie, M. : "Few differences in hot and cold executive functions in children and adolescents with combined and inattentive subtypes of ADHD", Child Neuropsychology: A Journal on Normal and Abnormal Development in Childhood and Adolescence", pp. 1-23, (2013).

[27] Sonuga-Barke, E. J. The dual pathway model of AD/HD: an elaboration of neurodevelopmental characteristics. Neuroscience and Biobehavioral Reviews, vol. 27 (7), 593-604, (2003). https://doi.org/10.1016/j.neubiorev.2003.08.005 
[28] Sonuga-Barke, E. J. Causal models of attention-deficit/hyperactivity disorder: from common simple deficits to multiple developmental pathways. Biological Psychiatry, 57 (11), 1231-1238, (2005). https://doi.org/10.1016/j.biopsych.2004.09.008

[29] Hovik K.T. Plessen K.J. Cavanna A.E. Skogli E.W. Andersen P.N. and Øie M. : "Cognition, Emotion and Behavior in Children with Tourette's Syndrome and Children with ADHD-Combined Subtype-A Two-Year Follow-Up Study, PLOS ONE, vol. 10 (12), (2015). https://doi.org/10.1371/journal.pone.0144874

[30] Blum K. Lih-Chuan Chen A. Braverman E. R. Comings D. E. Chen T. JH. Arcuri V. Blum S. H. Downs B. W. Waite R. L. Notaro A. Lubar J. Williams L. Prihoda T. J. Palomo T. and Oscar-Berman M. : "Attention-deficit-hyperactivity disorder and reward deficiency syndrome”, Neuropsychiatr Dis Treat, vol. 4 (5), 893-918, (2008).

[31] Bunford N. Evans S. W. and Wymbs F. : "ADHD and Emotion Dysregulation Among Children and Adolescents", Clin Child Fam Psychol Rev, vol. 18 (3), pp. 185-217, (2015). https://doi.org/10.1007/s10567-015-0187-5

[32] Posner M. I and Rothbart M. K. : “Attention, self-regulation and consciousness", vol. 353 (1377), The Royal Society, (1998).

\section{Authors}

A. Drigas is a Research Director at N.C.S.R. 'Demokritos', Institute of Informatics and Telecommunications, Telecoms Lab - Net Media Lab, Agia Paraskevi, 153 10, Athens, Greece (e-mail: dr@iit.demokritos.gr).

M. Karyotaki is a Phd candidate in cognitive skills with N.C.S.R. 'Demokritos', Institute of Informatics and Telecommunications, Telecoms Lab - Net Media Lab, Agia Paraskevi, 153 10, Athens, Greece (e-mail: karyotakimaria@gmail.com).

C. Skianis is an Associate Professor in the Department of Information and Communication Systems Engineering at the University of the Aegean, Karlovassi, Greece (e-mail:cskianis@aegean.gr).

Article submitted 23 December 2016. Published as resubmitted by the authors 27 January 2017. 